Article

\title{
Matrilin-2 Is Proteolytically Cleaved by ADAMTS-4 and ADAMTS-5
}

\author{
Zhengke Wang ${ }^{1,2, *}$, Junming Luo ${ }^{1}$, Satori Iwamoto ${ }^{2}$ and Qian Chen ${ }^{1, *}$
}

1 Cell and Molecular Biology Laboratory, Department of Orthopaedics, The Warren Alpert Medical School of Brown University, Rhode Island Hospital, Providence, RI 02903, USA

2 Department of Dermatology, Roger Williams Medical Center, Providence, RI 02908, USA

* Authors to whom correspondence should be addressed; E-Mails: zwang@rwmc.org (Z.W.); Qian_Chen@Brown.edu (Q.C.); Tel.: +1-401-456-2087 (Z.W.); +1-401-444-6604 (Q.C.); Fax: +1-401-456-5872 (Z.W.); +1-401-444-5872 (Q.C.).

Received: 8 April 2014; in revised form: 7 June 2014 / Accepted: 10 June 2014 /

Published: 23 June 2014

\begin{abstract}
Matrilin-2 is a widely distributed, oligomeric extracellular matrix protein that forms a filamentous network by binding to a variety of different extracellular matrix proteins. We found matrilin-2 proteolytic products in transfected cell lines in vitro and in mouse tissues in vivo. Two putative cleavage sites were identified in the unique domain of matrilin-2; the first site was located between $\mathrm{D}^{851}$ and $\mathrm{L}^{852}$ in the middle of the domain and the second, at the boundary with the coiled-coil domain at the C-terminus. Deletion of the entire unique domain eliminated the proteolysis of matrilin-2. While the first cleavage site was present in all matrilin-2 oligomers, the second cleavage site became apparent only in the matrilin-2 hetero-oligomers with matrilin-1 or matrilin-3. Analysis using a variety of extracellular protease inhibitors suggested that this proteolytic activity was derived from a member or several members of the ADAMTS family. Recombinant human ADAMTS-4 (aggrecanase-1) and ADAMTS-5 (aggrecanase-2), but not ADAMTS-1, cleaved recombinant matrilin-2, thereby yielding matrilin-2 proteolytic peptides at the predicted sizes. These results suggest that ADAMTS-4 and ADAMTS-5 may destabilize the filamentous network in the extracellular matrix by cleaving matrilin-2 in both homo-oligomers and hetero-oligomers.
\end{abstract}

Keywords: matrilin-2; proteolytic cleavage; ADAMTS-4; ADAMTS-5; oligomerization 


\section{Introduction}

Matrilins are a family of four non-collagenous extracellular matrix (ECM) proteins $[1,2]$. Among them, matrilin-1 [3] and matrilin-3 [4,5] are expressed mainly in cartilage, while matrilin-2 [6] and matrilin-4 [7,8] are widely distributed in many connective tissues [9]. Matrilin-2 (MATN2) is the largest and the most complex matrilin. It is made up by two vWFA-like domains flanked by ten EGF modules with a unique domain located between the vWFA2 and the coiled-coil domain at its C-terminus [6]. Matrilin-2 is widely distributed in extracellular matrices of many connective tissues $[9,10]$. Matrilin-2 can bind to collagen I and non-collagenous proteins, such as fibrillin-2, fibronectin, and laminin-1-nidogen-1 complexes [11]. Matrilin-2 is expressed by pre-myelinating Schwann cells during normal development. It increases neurite outgrowth of dorsal root ganglia (DRG) neurons and enhances the migration of embryonic DRG-derived Schwann cells [12]. In addition, matrilin-2 is synthesized by both keratinocytes and dermal fibroblasts in human skin, which is further processed by cell-associated proteases [13]. Matrilin-2 gene expression is regulated by BMP-7 and the transcription factor DeltaNp63, which is responsible for keratinocyte cell migration [14].

Extracellular proteinases are required for numerous developmental and homeostasis processes. Excessive degradation or accumulation of extracellular matrix macromolecules, as a result of malfunction of extracellular proteinases or their matrix substrates, leads to various human diseases. For example, degeneration and loss of extracellular matrix macromolecules from cartilage result in serious impairment of joint function. Extracellular proteinases include both matrix metalloproteinases (MMP), which are extensively characterized, and the ADAMTS family members. The ADAMTS proteases are extracellular matrix proteins with ADAM-like protease domain and matrix-binding thrombospondin type 1-repeat $[15,16]$. Among them, two cartilage aggrecanases, aggrecanase-1 (ADAMTS-4) and aggrecanase-2 (ADAMTS-5) are upregulated in human osteoarthritic (OA) cartilage. They are responsible for aggrecan degradation in the absence of other matrix metalloproteinases [17]. ADAMTS-4 is a glutamyl endopeptidase that preferentially cleaves Glu-Xaa bonds of the core protein of proteoglycans such as aggrecan, brevican, and versican [18,19]. This proteolytic process was thought to depend on the presence of glycosaminoglycans in the substrate [20,21].

Matrilins form a filamentous network to connect their ligands (including integrins [22], collagens [23,24], proteoglycans [25], and other non-collagenous glycoproteins) in the extracellular matrix [26]. All matrilins contain the following: one or two von Willebrand Factor (vWF) A domains, a variable number of epidermal growth factor (EGF) domains, and a coiled-coil domain at the $C$-terminal end [1]. The vWF A domain is responsible for the interaction of matrilins with their matrix ligands through a metal-ion dependent adhesion site [3]. The coiled-coil domain is responsible for oligomerization of matrilin subunits, which include homo-oligomerization, or hetero-oligomerization if two different matrilin molecules are expressed in the same tissue at the same time [27,28]. We previously found evidence for a proteolytic cleavage site between the vWF domain and the coiledcoil domain in matrilin-1 [29]. Proteolytic cleavage may decrease matrilins' ability to bind other extracellular matrix proteins when the hetero-oligomers are proteolyzed into fragments. It was recently shown that matrilin-3 [30] and matrilin-4 [31] are both cleaved by ADAMTS-4 in the hinge region at the specific Glu-Xaa bond. Physiological cleavage has been described for most members of the matrilin family members. Interestingly, an identified cleavage of matrilin-4 [31] at the N-terminus of 
the coiled-coil domain, is conserved throughout the matrilin family [29,30]. Our previous work had shown that two alternatively-spliced isoforms in the unique domain of matrilin-2 resulted in different matrilin-2 oligomers [32]. In this study, we analyzed matrilin-2 proteolytic processing and found matrilin-2 to be a substrate of aggrecanase-1 (ADAMTS-4) and aggrecanase-2 (ADAMTS-5). Two separate cleavage sites were identified in matrilin-2. One was located at the boundary of the unique domain with the coiled-coil domain as in other matrilin cleavage sites. The other cleavage site was a previously unreported site between the Asp and Leu residues in the middle of the unique domain.

\section{Results and Discussion}

\subsection{Proteolytic Processing of Matrilin-2}

To study the role of the unique domain of matrilin-2 in the proteolytic cleavage of matrilin-2, we cloned a mouse matrilin-2 cDNA (M2L) that contained the $C$-terminal half of the molecule including the vWFA2 domain, the unique domain, and the coiled-coil domain. After the M2L cDNA was transfected into Cos-1 cells, secreted recombinant MATN2 (rMATN2) peptides in the conditioned medium were affinity purified using the antibody against the FLAG tag at the N-terminus of rMATN2. In addition to the full length M2L peptide at the expected molecular weight of 40kDa, another smaller peptide at $23 \mathrm{kDa}$ was detected (Figure 1A). This suggested that the $40 \mathrm{kDa}$ full-length rMATN2 was cleaved to yield an $N$-terminal peptide of $23 \mathrm{kDa}$. This cleavage product was detected in both Cos- 1 cells and MCT chondrocytes cultures (Figure 1B). Thus, both kidney and cartilage derived cells contain the proteolytic activity. Interestingly, expression of rMATN2 alone exhibited a different proteolytic pattern than co-expression of rMATN2 with other matrilins. While single transfection of M2L generated a $23 \mathrm{kDa} N$-terminal MATN2 proteolytic fragment, its co-transfection with a MATN1 or MATN3 cDNA generated an additional $N$-terminal $25 \mathrm{kDa}$ matrilin-2 fragment (Figure 1C). Thus, co-expression of matrilin-2 with other matrilins affects the processing of matrilin-2. To determine whether such proteolysis involved matrix metalloproteinase, we added EDTA, a matrix metalloproteinase inhibitor (Figure 1D), and actinonin, which inhibits $100 \%$ of aggrecanase and $22.5 \% \mathrm{MMP}$ at $100 \mathrm{M}$ [33] into the medium (Figure 1E). Both compounds inhibited rMATN2 proteolysis (Figure 1D,E).

\subsection{Matrilin-2 Was Cleaved by ADAMTS-4 and ADAMTS-5 but Not ADAMTS-1}

To determine whether aggrecanases were responsible for proteolysis of matrilin-2, conditioned medium from M2L transfected cells was harvested and incubated with purified recombinant human ADAMTS-4 (aggrecanase-1) at $37{ }^{\circ} \mathrm{C}$ for $24 \mathrm{~h}$. Incubation with ADAMTS-4 greatly increased the yield of the $23 \mathrm{kDa}$ cleavage product (Figure 2A right). ADAMTS-5 incubation also significantly increased the $23 \mathrm{kDa}$ rMATN2 N-terminal cleavage peptide (Figure 2B). However, ADAMTS-1 incubation did not increase the yield of matrilin-2 cleavage peptide (Figure 2C). Furthermore, ADAMTS-4 proteolysis of rMATN2 was completely inhibited by $5 \mathrm{mM}$ EDTA, a matrix metalloproteinase inhibitor (Figure 2D) and by $100 \mu \mathrm{M}$ actinonin (Figure 2E). Therefore, we concluded that aggrecanases were involved in rMATN2 proteolysis. Furthermore, western blot analyses indicated that proteolysis of matrilin-2 was dependent on the concentration of ADAMTS-4 (Figure 2F). 
Figure 1. Matrilin-2 Proteolysis in vitro. (A) Purified M2L peptides visualized on a 4\%-15\% SDS-PAGE gradient gel. Conditioned medium from M2L transfected COS-1 cell line was affinity purified with an anti-FLAG monoclonal antibody, separated by electrophoresis and visualized by Coomassie blue staining. Mouse normal IgG indicates an control experiemnt in which IgG replaced anti-FLAG monoclonal antibody. Non-specific IgG heavy chain (IgGH) and IgG light chain (IgGL) were indicated; (B) Western blot analysis of M2L peptides from the conditioned medium collected from M2L transfected monkey kidney epithilial COS-1 cells and mouse chondrocyte MCT cells, respectively. Conditioned medium was subjected to electrophoresis and followed by western blot analysis with an anti-FLAG antibody; (C) Different proteolytic cleavage patterns of matrilin-2 from single or co-transfections of matrilins. Conditioned medium from Cos-1 cells transfected with M2L or co-transfection of M2L with either matrilin-1or matrilin-3 was analyzed by electrophoresis on an 4\%-15\% SDS-PAGE gel followed by western blot with an anti-FLAG antibody. Note the apperance of a doublet of cleavage products in cotransfection samples; (D) Western blot analysis of matrilin-2 peptides from the conditioned medium of Cos- 1 cells co-transfected with M2L and matrilin-1, or with M2L and matrilin-3. Different concentrations of EDTA were added into culture medium for $72 \mathrm{~h}$. The conditioned medium was collected and subjected to SDS-PAGE electrophoresis followed by western blotting using an anti-FLAG polyclonal antibody; (E) Actinonin inhibit matrilin-2 cleavage. Western blot analysis of matrilin-2 peptides from the conditioned medium of Cos- 1 cells co-transfected with M2L and matrilin-1, or with M2L and matrilin-3. Different concentrations of actinonin were added into culture medium for $72 \mathrm{~h}$. The conditioned medium was collected and subjected to SDS-PAGE electrophoresis followed by western blotting using an anti-FLAG polyclonal antibody.

A

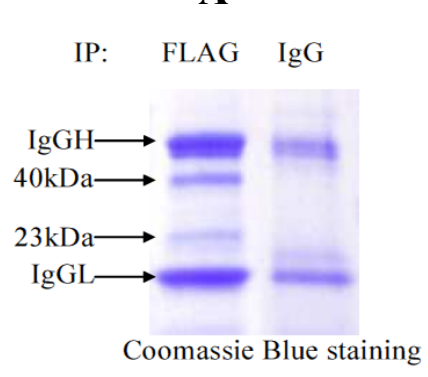

D

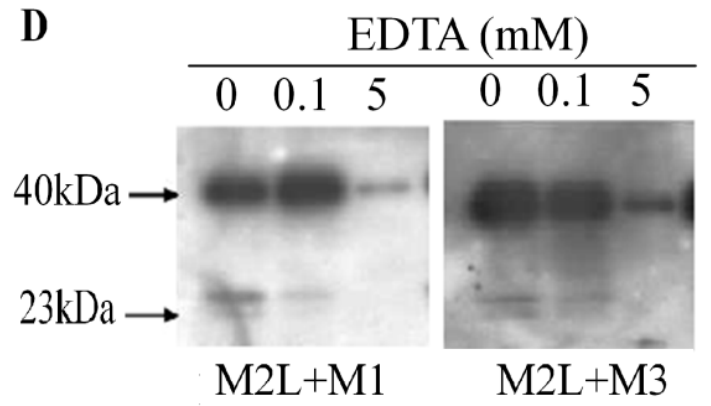

B COS-1 MCT

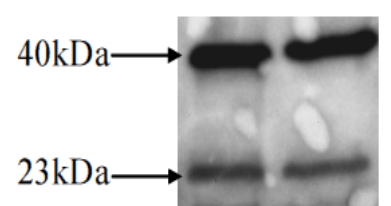

WB:Ab Anti-FLAG pAb
C

M2L M2L M2L $+\mathrm{M} 1+\mathrm{M} 3$

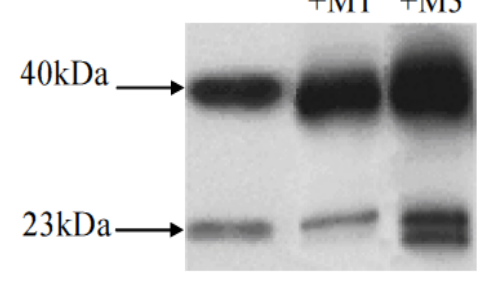

E actinonin $(\mu \mathrm{M})$

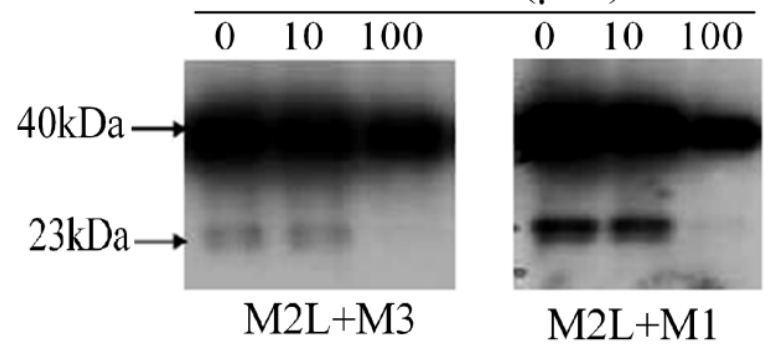


Figure 2. Matrilin-2 was cleaved by purified human ADAMTS-4 and ADAMTS-5 specifically. Matrilin-2 peptides were incubated with or without purified recombinant ADAMTS-4 (A), ADAMTS-5 (B) and ADAMTS-1 (C). Western blot analysis of the conditioned medium of Cos-1 cells was performed using an anti-FLAG polyclonal antibody. EDTA (D) or actinonin (E) was added into cell culture. These conditioned media were incubated with or without purified recombinant ADAMTS-4 followed by western blot analysis with an anti-FLAG polyclonal antibody. (F) ADAMTS-4 cleavage of matrilin-2 was dosage dependent. Purified M2L (1 ng) in $10 \mu \mathrm{L}$ reaction buffer was incubated with purified human recombinant ADAMTS-4 $(5 \mu \mathrm{g} / 25 \mu \mathrm{L})$ at the contents of 0 , $1,2,4,8,12$, and $16 \mu \mathrm{L}$ at $37^{\circ} \mathrm{C}$ for $24 \mathrm{~h}$, followed by western blot analysis using anti-flag poly clonal antiboby.

A

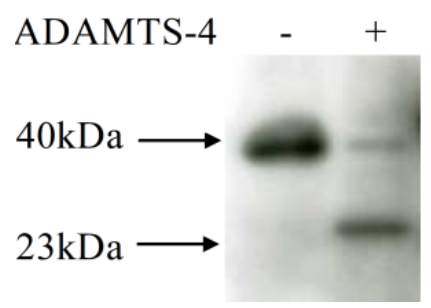

D

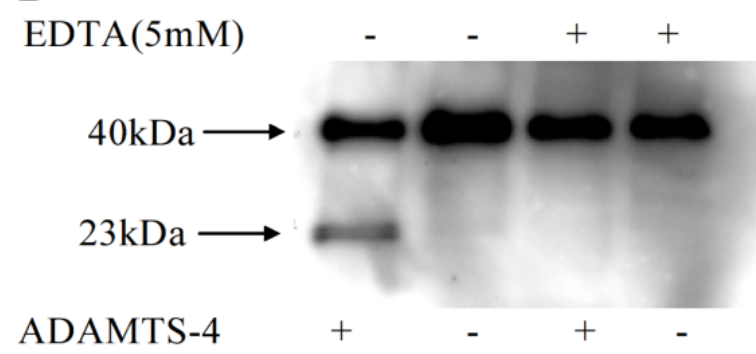

C
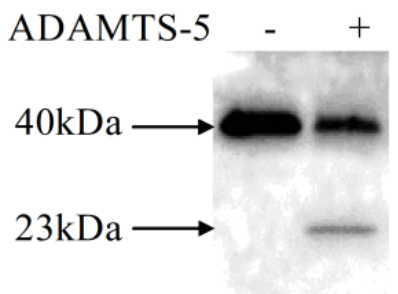

$\mathbf{E}$

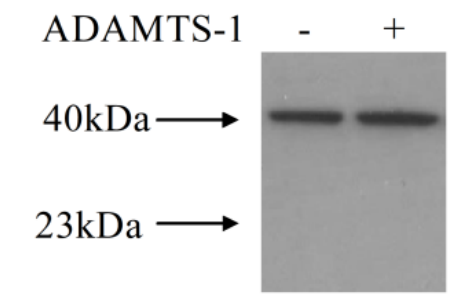

$\operatorname{Actinonin}(\mu \mathrm{M})$

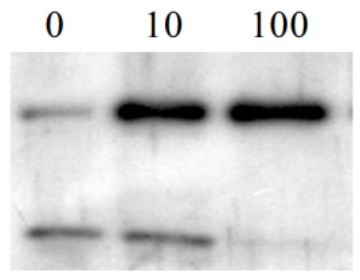

ADAMTS- $4+\quad+\quad+$

(F)

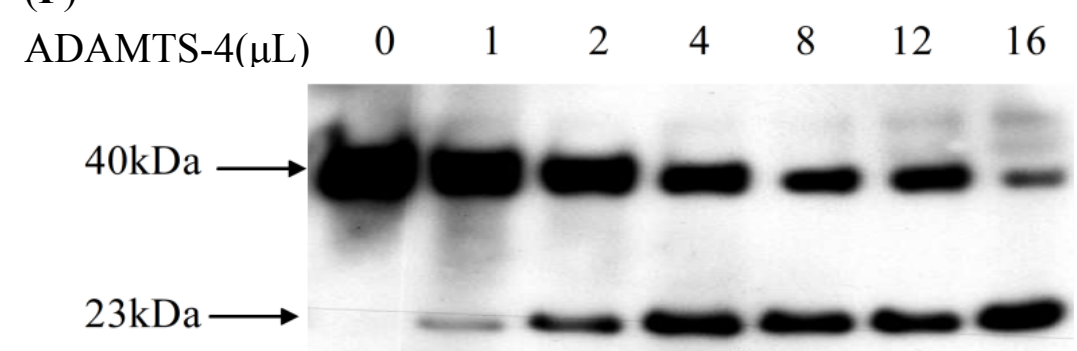

\subsection{Identification of the Proteolytic Site in the Unique Domain of Matrilin-2}

To identify the proteolytic cleavage sites within the unique domain (Figure 3A), we generated a series of mouse matrilin-2 cDNAs containing various deletions and mutations in the unique domain of matrilin-2 (Figure 3B). 
Figure 3. Diagram of matrilin-2 and its cDNA constructs. (A) A schematic diagram of matrilin-2 and the amino acid sequence of the unique domain. Alternative splicing region and the antigenic peptide for generation of anti-matrilin-2 antibody were indicated. The numbers correspond to different truncation sites within the domain. (B) Matrilin-2 cDNA constructs with deletions and mutations in the unique domain. M2L(Long) contains the entire unique domain ( $\mathrm{A}^{838}$ to $\mathrm{E}^{911}$ ). M2S(Short) contains the short form of the unique domain without the alternative splicing region $\left(\mathrm{E}^{861}\right.$ to $\mathrm{A}^{879}$ ). $\mathrm{M} 2 \mathrm{D}$ (Deleted) has the entire unique domain deleted. $\mathrm{M} 2 \mathrm{~d} 1, \mathrm{M} 2 \mathrm{~d} 2, \mathrm{M} 2 \mathrm{~d} 3$ were matrilin-2 truncated products in which the stop codon was added after $\mathrm{E}^{911}$ (position 1), $\mathrm{E}^{888}$ (position 2), $\mathrm{E}^{861}$ (position 3) in $\mathrm{M} 2 \mathrm{~L}$ respectively. Position 4 between $\mathrm{D}$ and $\mathrm{L}$ is the putative unique ADAMTS cleavage site in matrilin-2, while position 1 between $E^{911}$ and $S^{912}$ is the second putative ADAMTS cleavage site, similar to the sites in other matrilins.

A

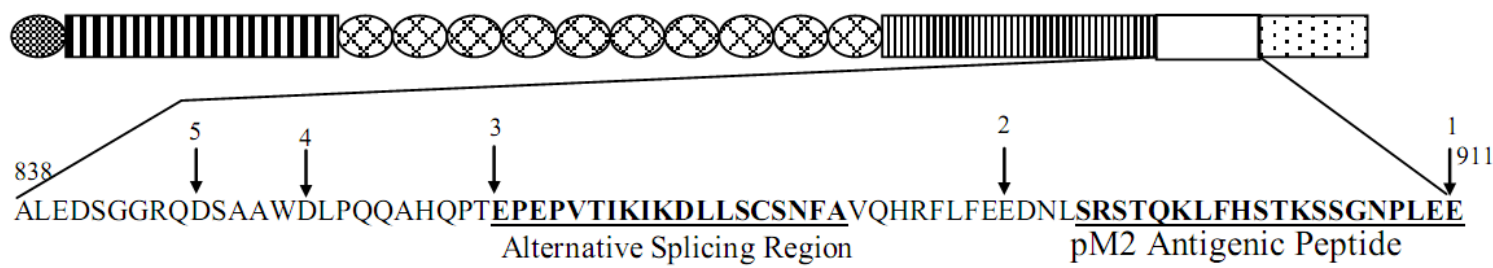

B

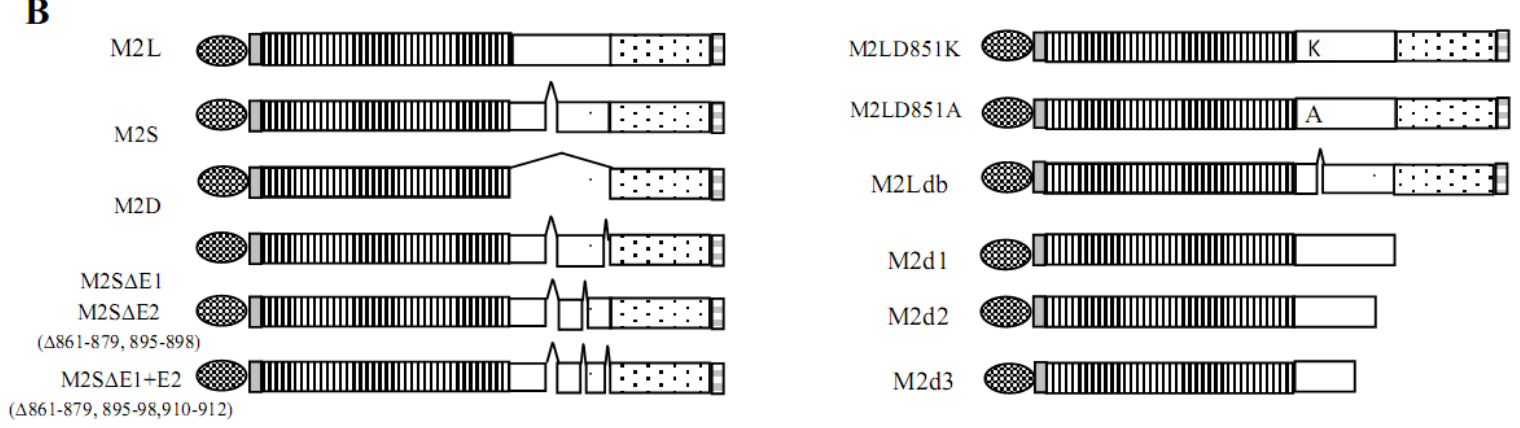

\begin{tabular}{|llllllll|}
\hline$\otimes$ & Signal Peptide & III & VWFA1 & $\square$ & Unique & $\square$ & FLAG tag \\
$\otimes$ & EGF & 盂 & VWFA2 & $\square$ & Coiled-coil & 貝 & His tag \\
\hline
\end{tabular}

To determine whether the proteolytic cleavage sites were located in the unique domain, we transfected M2L, M2S, and M2D into Cos-1 cells. While transfection of M2L that contained the entire unique domain resulted in the $23 \mathrm{kD}$ cleavage product, transfection of $\mathrm{M} 2 \mathrm{~S}$ with a 19 amino acid deletion in the unique domain produced a low level of cleavage product. Transfection of M2D, which lacked the entire unique domain, produced no cleavage product (Figure 4A). These results suggested that the cleavage sites were located within the unique domain.

Because the conserved ADAMTS cleavage site in aggrecan, bevican, and matrilin-3 occurred after the glutamic acid residue, we systematically deleted glutamic acid residues within the unique domain. While the wild type matrilin-2 co-transfection with matrilin-3 produced two cleavage products at 23 and $25 \mathrm{kDa}$, the M2L $\Delta \mathrm{E} 1$ mutant which lacked the glutamic acid-glutamic acid-aspartic acid residues at position 910-912, produced only the $23 \mathrm{kDa}$ product (Figure 4B), suggesting that these three amino acid residues were required for the ADAMTS generation of the $25 \mathrm{kDa}$, but not the $23 \mathrm{kDa}$ cleavage 
peptide. Furthermore, ADAMTS-4 incubation produced a $23 \mathrm{kDa}$ cleavage peptide in the MATN2 mutants lacking one or both glutamic acids (E1 or E2). This suggested the existence of a second (previously unreported) ADAMTS-4 cleavage site in the unique domain, and one that did not follow a glutamic acid residue.

Figure 4. Identification of matrilin-2 proteolytic cleavage sites. (A) The unique domain is required for matrilin-2 proteolysis. Western blot analysis of matrilin-2 products in the conditioned medium collected from Cos-1 cells co-transfected with M2L and M1 (matrilin-1), M2S and M1, or M2D and M1 respectively, and western blot with an anti-FLAG antibody; (B) The EES site in the unique domain of matrilin-2 is involved in the proteolysis by ADAMTS-4. Wild type M2L and M2LAE1 (EES deletion) were co-transfected with M3 (matrilin-3) cDNA in Cos-1 cells. Condidtioned medium was incubated with recombinant human ADAMTS-4 before western blot analysis with an anti-FLAG antibody; (C) ADAMTS-4 cleaves matrilin-2 mutants harboring glutamate acid deletions in the unique domain, including M2S (WT), M2S $\Delta \mathrm{E} 1, \mathrm{M} 2 \mathrm{~S} \Delta \mathrm{E} 2$, and M2S $\Delta \mathrm{E} 1+2$. M2D was used as a negative control. Western blot analysis was performed with the conditioned medium collected from Cos-1 cells after treatment with recombinant human ADAMTS-4; (D) Western blot analysis of matrilin-2 truncation products $\mathrm{M} 2 \mathrm{~d} 1, \mathrm{M} 2 \mathrm{~d} 2$ and $\mathrm{M} 2 \mathrm{~d} 3$ in comparison to the $23 \mathrm{kDa}$ M2L ADAMTS-4 cleavage product; (E) Recombinant M2L was purified from the conditioned medium of M2L transfected Cos- 1 cells by Ni-NTA procedure and visualized by Coomassie blue staining; (F) Mass spectrometry analysis of purified M2L product incubated with or without recombinant human ADAMTS-4. Molecular mass of M2L cleavage product is indicated; $(\mathbf{G})$ and $(\mathbf{H})$ Matrilin-2 cleavage site mutants are resistant to ADAMTS-4 proteolysis. Wild-type matrilin-2, site deletion mutant $(\mathrm{db})$ or point mutation (D851K, D851A) mutant were incubated with purified recombinant human ADAMTS-4 followed by western blot analysis with anti-FLAG polyclonal antiboby.

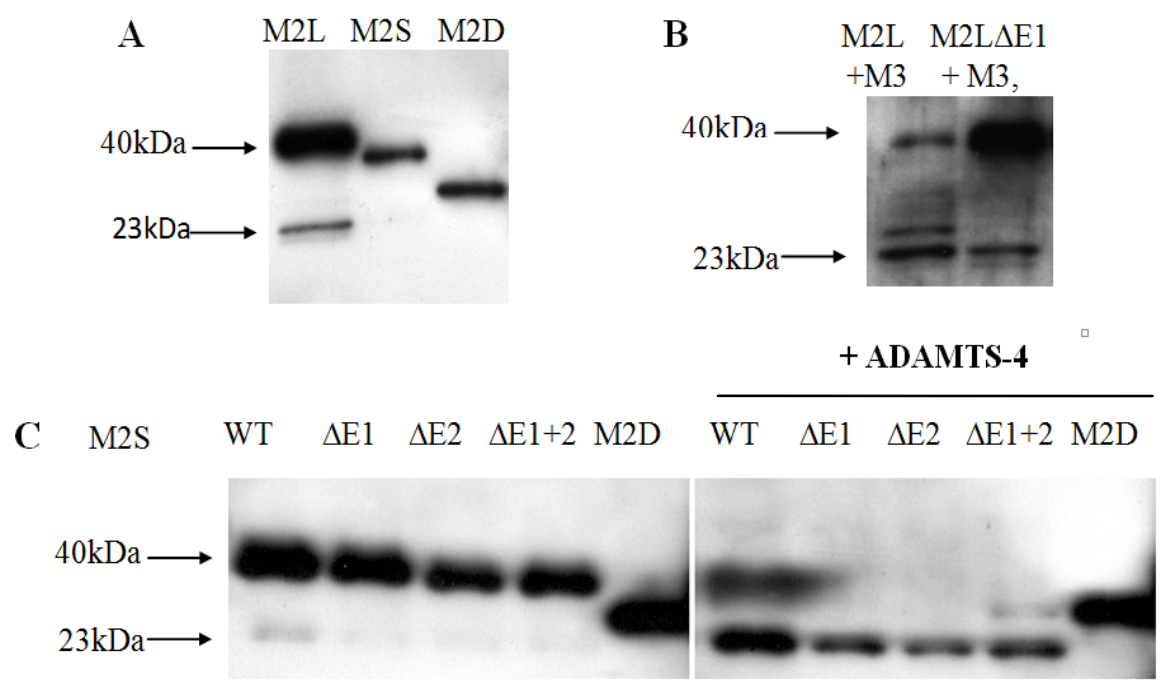


Figure 4. Cont.

D

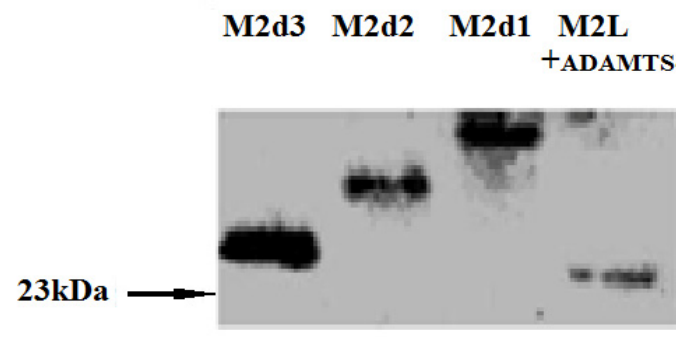

$\mathbf{E}$

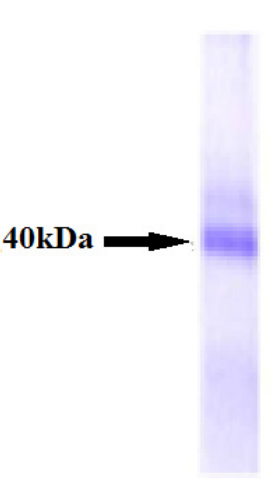

Proteolytic

cleavage site

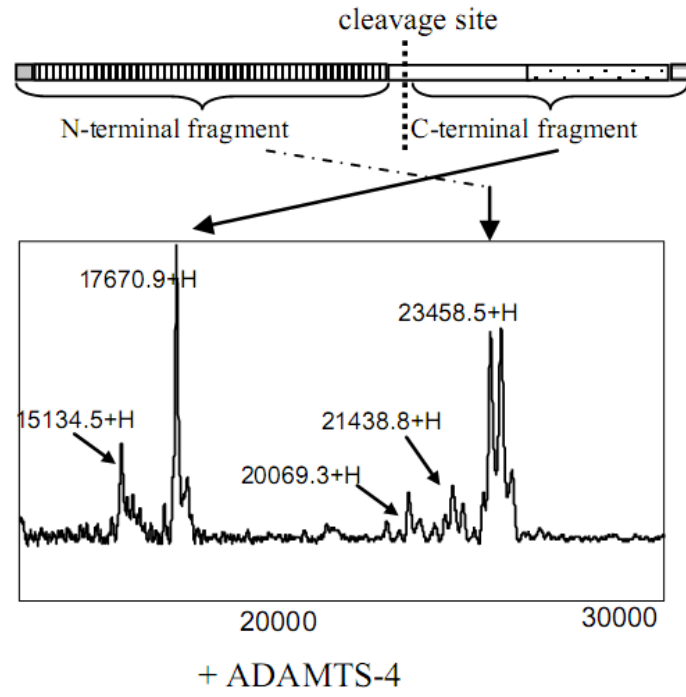

H

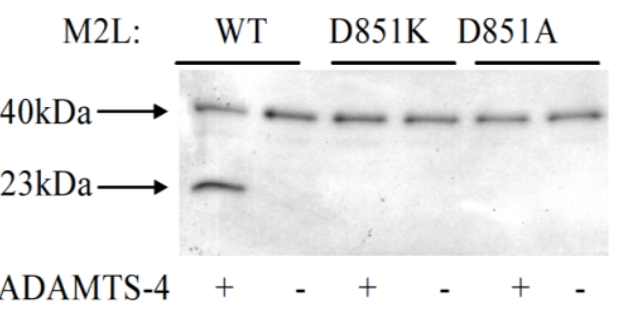

To locate this novel ADAMTS-4 cleavage site, we created a set of truncated matrilin-2 constructs by adding the stop codon at three specific sites within the unique domain (Figure 3B). M2d1 truncated the protein at the $C$-terminal end of the unique domain. M2d2 truncated the protein at the C-terminus of the alternative splicing region. And $\mathrm{M} 2 \mathrm{~d} 3$ truncated the protein at the $\mathrm{N}$-terminus of the alternativesplicing region. Western blot analyses indicated that the ADAMTS-4 cleavage product was smaller than all three truncated products (Figure 4D), suggesting that the cleavage site was located within the first 25 amino acids of the N-terminal region before the alternative splicing region in the unique domain.

To more precisely identify the cleavage site, we affinity-purified the full-length $40 \mathrm{kDa}$ rMATN2 from the conditioned medium (Figure 4E). Incubation of purified rMATN2 with recombinant ADAMTS-4 at $37{ }^{\circ} \mathrm{C}$ for $24 \mathrm{~h}$ resulted in two peaks by SELDI mass spectrometry, representing matrilin-2 peptides of 17,670.9 Dalton and 23,658.5 Dalton (Figure 4F). Based on the calculated 
molecular weights, we calculated the matrilin- 2 proteolytic cleavage site to be between the $\mathrm{D}^{851}$ and $\mathrm{L}^{852}$ residues (Figure 4D). To further confirm the matrilin-2 cleavage site, we performed deletion and point mutation analyses of the putative cleavage site. While ADAMTS-4 could cleave the wild type rMATN2, it could not cleave the mutant M2Ldb $\left(\Delta \mathrm{W}^{850} \mathrm{DLP}^{853}\right)$ in which the cleavage site was deleted (Figure 4G). Furthermore, ADAMTS-4 could not cleave the matrilin-2 peptides harboring point mutations of the putative cleavage site, including M2LD851A (in which changed the negatively charged amino acid aspartic acid had been changed to the non-charged valine) and M2LD851K (in which the negatively charged amino acid aspartic acid had been changed to the positively charged amino acid lysine) (Figure 4G). In contrast, the point mutations of glutamic acid residues in the middle of the unique domain did not change the matrilin-2 cleavage pattern (Figure 4C).

Of the two cleavage sites, one follows a glutamic acid residue and the other does not. A glutamic acid residue commonly precedes the cleavage sites of many glycosaminoglycans (GAG) proteins. For example, ADAMTS-4 and ADAMTS-5 cleave the following substrates after the glutamic acid residue: Aggrecan $\left(\mathrm{Glu}^{373} / \mathrm{Ala}^{374}\right.$, Glu ${ }^{1545} / \mathrm{Gly}^{1546}$, Glu $^{1714} / \mathrm{Gly}^{1715}{ }$ Glu $^{1819} / \mathrm{Ala}^{1820}$, Glu $\left.^{1919} / \mathrm{Leu}^{1920}\right)[15,19,34]$, versican $\left(\mathrm{Glu}^{441} / \mathrm{Ala}^{442}\right)$ [35], and brevican $\left(\mathrm{Glu}^{395} / \mathrm{Ser}^{396}\right)$ [36]. Less commonly, ADAMTS-4 and ADAMTS-5 also cleave substrates at other sites. For example, ADAMTS-4 and ADAMTS-5 cleave $\alpha_{2}$-macroglobulin at the $\mathrm{Met}^{690} / \mathrm{Gly}^{691}$ [37] and aggrecan at $\mathrm{Asn}^{341} / \mathrm{Phe}^{342}$ [38].

ADAMTS-4 and ADAMTS-5 cleave not only matrilin-2 but also other members of the matrilin family. In addition, Matrilin-4 has been reported to be cleaved at a site between the vWFA2 domain and the coiled-coil domain [7], similar to the location of the proteolytic site in matrilin-2 identified in this study. Matrilin-1 also possesses a proteolytic cleavage site between the glutamic acid and glycine residues in the hinge region [29]. All four matrilin members possess a proteolytic cleavage site after glutamic acid (matrilin-3 at $\mathrm{Glu}^{435} / \mathrm{Ala}^{436}$ [30], and matrilin-4 at Glu ${ }^{570}$ $\mathrm{Glu}^{571} / \mathrm{G}^{572}$ ) [31]. However, matrilin-2 may be unique in that it possesses two proteolytic cleavage sites, one of which follows a glutamic acid residue and the other of which follows an aspartic acid residue.

\subsection{Matrilin-2 Proteolytic Cleavage in Vivo and the Proteolytic Susceptibility of the Splicing Variants}

To determine whether the processing also occurred in vivo, we generated a polyclonal antibody (pM2) against a synthetic peptide from the unique domain of mouse matrilin-2 (Figure 3A). Since this antigenic peptide is located at the $C$-terminal side to the $\mathrm{D}^{851} / \mathrm{L}^{852}$ cleavage site, we reasoned that this antibody should detect a $17 \mathrm{kDa}$ (the difference in size between the $40 \mathrm{kDa}$ full length matrilin protein and the $23 \mathrm{kDa}$-terminal fragment) $C$-terminal MATN-2 proteolytic product resulted from matrilin-2 proteolysis. We analyzed tissue extracts from the mouse urine bladder that were known to express matrilin-2 [39] with western blot using the pM2 antibody. A $17 \mathrm{kDa}$ matrilin-2 proteolytic fragment was indeed detected in addition to the full-length MATN2 from tissue extracts (Figure 5A). Thus, we concluded that proteolytic processing of MATN2 also occurred in vivo. Using an antibody against the His-tag at the $\mathrm{C}$ terminal end of rMATN2, we detected a $17 \mathrm{kDa}$ product with the recombinant MATN2 (Figure 5B), suggesting that the in vivo and in vitro processing of matrilin-2 involved the same proteolytic site. 
Figure 5. Matrilin-2 proteolytic cleavage in vivo and the proteolysis susceptibility of the splicing variants. (A) Matrilin-2 proteolytic cleavage product in mouse urine bladder. Urine bladder from newborn mice was homogenized and analyzed by electrophoresis in a 4\%-15\% SDS-PAGE gel under reducing conditions, followed by western blot analysis using a polyclonal antibody pM2 against matrilin-2. A full length $113 \mathrm{kDa}$ matrilin-2 monomer and the $17 \mathrm{kDa} C$-terminal cleavage product were indicated; (B) Western blot analysis of $C$-terminal matrilin-2 proteolytic cleavage product. M2L transfected cells were incubated with ADAMTS-4 for $24 \mathrm{~h}$. Electrophoresis was performed in a $4 \%-15 \%$ SDS-PAGE gel under reduced conditions, followed by western blot analysis using a monoclonal antibody against the His tag at the C-terminus; (C) M2L or M2S transfected cells were incubated with ADAMTS-4 for $24 \mathrm{~h}$. Electrophoresis was performed in a $4 \%-15 \%$ SDS-PAGE gel under non-reduced conditions, followed by western blot analysis using a monoclonal antibody against the FLAG tag at the N-terminus.
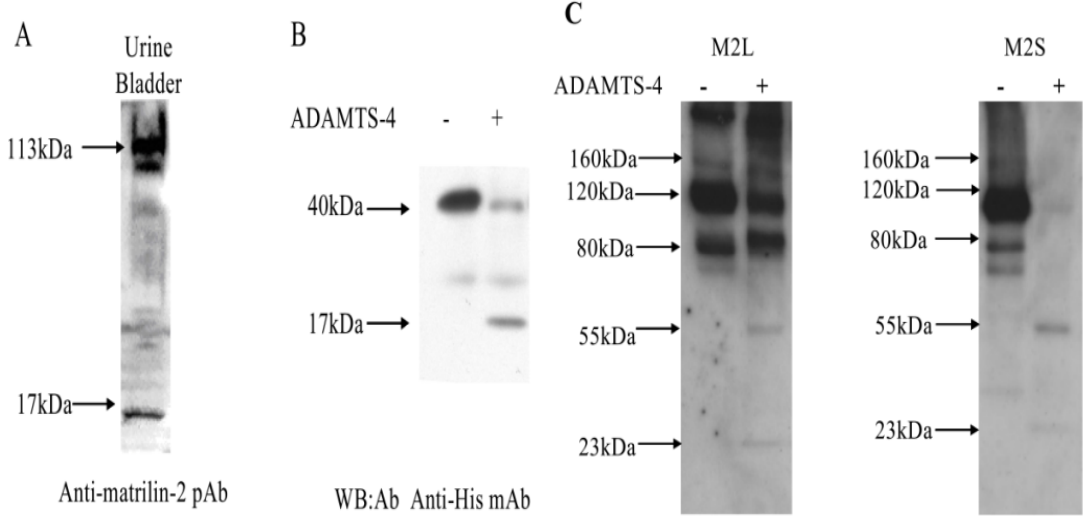

The long and short splice variants of matrilin-2 mRNA were expressed in all mouse skeletal and non-skeletal tissues [32]. To determine the susceptibility of the MATN2 splice variant peptide products to ADAMTS-4 proteolysis, we incubated the protein products of M2L and M2S with ADAMTS-4 (Figure 5C). Prior to ADAMTS-4 proteolysis, M2L protein products existed as dimers $(80 \mathrm{kDa})$, trimers $(120 \mathrm{kDa})$, tetramers $(160 \mathrm{kDa})$, or multimers, and M2S protein products existed as dimers $(74 \mathrm{kDa})$, trimers $(111 \mathrm{kDa})$, tetramers $(148 \mathrm{kDa})$, or multimers. After ADAMTS-4 proteolysis, the N-terminal cleavage peptide of $23 \mathrm{kDa}$ appeared in both splicing variant peptide products as well as with the full length peptide linked with the $C$-terminal cleavage peptide $(57 \mathrm{kDa}(=40+17)$ in $\mathrm{M} 2 \mathrm{~L}$, and $54 \mathrm{kDa}(=37+17)$ in M2S) (Figure 5C). Most strikingly, M2S was much more extensively cleaved than M2L, suggesting the long splice variant peptide product of MATN2 was more resistant to ADAMTS-4 proteolysis than the short splicing variant product.

\section{Experimental}

\subsection{Cloning and Construction of Matrilin-2 cDNAs}

Mouse matrilin-2 cDNA was cloned by RT-PCR from RNA isolated from sternal cartilage of newborn mice. Total RNA was isolated using the RNeasy kit (Qiagen, GmbH, Hilden, Germany). RT-PCR of matrilin-2 mRNA was performed using Titan One Tube RT-PCR system (Boehringer 
Mannheim, Indianapolis, IN, USA) according to manufacturer's instructions. Two step-PCR was used in the same tube under the following conditions: $94{ }^{\circ} \mathrm{C}$ for $30 \mathrm{~s}, 50{ }^{\circ} \mathrm{C}$ for $30 \mathrm{~s}$, and $68{ }^{\circ} \mathrm{C}$ for $1.5 \mathrm{~min}$ for 10 cycles. Then, the annealing temperature was raised to $55^{\circ} \mathrm{C}$ for another 20 cycles. The matrilin2 cDNA as well as cDNAs encoding chicken matrilin-1 and -3 from previous studies $[28,40]$ were cloned into an expression vector pcDNA3.1/V5-His (Invitrogen, Carlsbad, CA, USA). Genetic engineering including the addition of a N-terminus FLAG tag, truncated mutations (M2Ld1, M2Ld2, $\mathrm{M} 2 \mathrm{Ld} 3$ ), deletion mutations (M2S $\Delta \mathrm{E} 1, \mathrm{M} 2 \mathrm{~S} \Delta \mathrm{E} 2, \mathrm{M} 2 \mathrm{~S} \Delta \mathrm{E} 1+\mathrm{E} 2, \mathrm{M} 2 \mathrm{Ldb}$ ), and point mutations (D851A, D851K) within the unique domain (Figure 1) was performed by overlapping PCR with the described primer sets (Table 1). The modified cDNAs were cloned between the Xho I and Not I restriction sites of pcDNA3.1. All constructs were confirmed by DNA sequencing.

Table 1. A list of primers for generating matrilin-2 cDNA constructs.

\begin{tabular}{|c|c|c|}
\hline Primers & Primer Sequences $\left(5^{\prime}->3^{\prime}\right)$ & PCR Purpose \\
\hline $\mathrm{T} 7$ & T AATACGACTCACTATAGGG & \multirow{2}{*}{ Cloning into pcDNA3.1/V5-His } \\
\hline BGH & CTAGAAGGCAACAGTCGAGG & \\
\hline Mat2flgas & GCATCTTTTGTCATCATCGTCCTT & \multirow{2}{*}{ Adding a FLAG tag in matrilin-2 } \\
\hline Mat2flgs & GATGACAAAAAGAGATGCACTGAAGGC & \\
\hline M2d1R & CGCTCGAGTCATTCTTCCAAAGGGTTCC & Creating M2d1 deletion mutant \\
\hline $\mathrm{M} 2 \mathrm{~d} 2 \mathrm{R}$ & CGCTCGAGTCATTCTTCAAACAGGAATC & Creating $\mathrm{M} 2 \mathrm{~d} 2$ deletion mutant \\
\hline $\mathrm{M} 2 \mathrm{~d} 3 \mathrm{R}$ & CGCTCGAGTCATTCTGTTGGCTGGTGGC & Creating M2d3 deletion mutant \\
\hline $\mathrm{M} 2 \mathrm{Ldb} 2 \mathrm{~F}$ & GACTCAGCAGCACAGCAGGCCCACCA & \multirow{2}{*}{ Creating M2Ldb mutant deleted 850WDLP853 } \\
\hline M2Ldb2R & GTGGGCCTGCTGTGCTGCTGAGTCCT & \\
\hline M2LD851KF & AGCATGGAAAGCTGCCACA & \multirow{2}{*}{ Creating M2LD851K mutant } \\
\hline M2LD851KR & TGTGGCAGCTTCCATGCT & \\
\hline M2LD851AF & AGCATGGGCCCTGCCACAGCAG & \multirow{2}{*}{ Creating M2LD851A mutant } \\
\hline M2LD851AR & CCTGCTGTGGCAGGGCCCATGCT & \\
\hline DETA3F & TTCCTGTTTAATCTTTCACGGTCTACACA & \multirow{3}{*}{$\begin{array}{l}\text { M2S } \Delta \text { E2 }(\Delta \text { Glu887 GluAsp889), } \\
\text { M2S } \Delta \text { E1 + E2( } \Delta \text { Glu887 GluAsp889, } \Delta \text { Glu910 } \\
\text { GluSer912) }\end{array}$} \\
\hline DFTA $3 R$ & GAAAAGTTAAACAGGAATCTATGTTGCA & \\
\hline & & \\
\hline DETA4F & CCCTTTGCAGGACCAATGCAAATGTGA & \multirow{3}{*}{$\begin{array}{l}\text { Creating M2S } \Delta \text { E1 ( } \Delta \text { Glu910 GluSer912), } \\
\text { M2S } \Delta \text { E1 + E2 }(\Delta \text { Glu887 GluAsp889, } \Delta \text { Glu910 } \\
\text { GluSer912) }\end{array}$} \\
\hline DFTA4R & GTCCTGCAAAGGGTTTCCTGAAGATTT & \\
\hline & & \\
\hline
\end{tabular}

\subsection{Transfection of Matrilin cDNAs}

cDNA constructs of matrilin-3 and -1 were transfected into COS-1 cells or MCT chondrocytes [41] using LIPOFECTAMINE 2000 (Life Technology, Rockville, MD, USA) according to the manufacturer's instructions. Briefly, COS-1 cells or MCT chondrocytes were trypsinized and counted. Each $60 \mathrm{~mm}$ plate was seeded with $6 \times 10^{5}$ cells. They were incubated at 37 degrees $\mathrm{C}$ overnight in DMEM (Gibco, Life Technologies, Grand Island, NY, USA) supplied with $10 \%$ FBS (Life technology) to allow the cells to reach $70 \%$ confluence. The following day, the cells were rinsed with DMEM and subjected to a DNA/LIPOFECTAMINE 2000 (Life Technology) mix for $5 \mathrm{~h}$. For single transfections, $5 \mu \mathrm{g}$ of DNA were used. For co-transfections, $4 \mu \mathrm{g}$ DNA of each of the constructs were used. The DNA/LIPOFECTAMINE 2000 mixture was aspirated and replaced with $3 \mathrm{~mL}$ DMEM 
supplied with $1 \%$ FBS. The media from transfected cell culture was collected at $72 \mathrm{~h}$ after transfection. Cells were lysed on ice for 10 minin a lysis buffer as previously described [28]. Cell lysates were centrifuged at $4{ }^{\circ} \mathrm{C}$ for $10 \mathrm{~min}$. Supernatant of the cell lysate, as well as of the conditioned medium, was analyzed using western blots. Some transfected cells were treated with EDTA, or actinonin at indicated concentrations for $48 \mathrm{~h}$ before the collection of the conditioned medium.

\subsection{SDS-Polyacrylamide Gel Electrophoresis and Western Blot}

Western blot analysis was performed with collected conditioned medium, with cell lysates from transfected cell cultures, or with urine bladder tissue extracts from new born mice as described previously [42]. Equal amounts of protein were loaded in each lane after quantification of the protein in the medium samples by bicinchoninic acid assay (BCA assay). For non-reducing conditions, collected samples were mixed with standard $2 \times$ SDS gel-loading buffer [28]. For reducing conditions, the loading buffer contains 5\% $\beta$-mercaptoethanol and 0.05 M DTT. Samples were boiled for $10 \mathrm{~min}$ before being loaded onto $10 \%$ SDS-PAGE gels or 4\%-20\% gradient gels. After electrophoresis, proteins were transferred onto Immobilon-PVDF membrane (Millipore Corp., Bedford, MA, USA) in $6 \mathrm{mM}$ Tris, $192 \mathrm{mM}$ glycine, and 15\% methanol. The membranes were blocked in 2\% bovine serum albumin fraction V (Sigma Co., St. Louis, MO, USA) in PBS for $30 \mathrm{~min}$ and then probed with antibodies.

The primary antibodies included a monoclonal antibody against the His tag (diluted 1:5000) (Invitrogen) and a monoclonal antibody against FLAG (diluted 1:1000) (Affinity BioReagents, Golden, CO, US). To generate a polyclonal antibody against matrilin-2, a peptide encoding 20 amino acids (SRSTQKLFHSTKSSGNPLEE) from the unique domain of mouse matrilin-2 was synthesized. The antibody was raised by immunizing rabbits with the synthetic peptide followed by affinity purification. Horseradish peroxidase conjugated goat anti-mouse or goat anti-rabbit $\operatorname{IgG}(\mathrm{H}+\mathrm{L})$ (Bio-Rad Laboratories, Melville, NY, USA), diluted 1:3000, was used as a secondary antibody. Visualization of immunoreactive proteins was achieved using the ECL Western blotting detection reagents (Amersham Corp., Heights, IL, USA) and exposing the membrane to Kodak X-Omat AR film. Molecular weights of the immunoreactive proteins were determined against two different sets of protein marker ladders.

\subsection{In Vitro Analysis of Matrilin-2 Proteolysis}

$12 \mu \mathrm{L}$ conditioned medium from matrilin-2 transfected cell culture was incubated with $0.8 \mu \mathrm{L}$ purified recombinant human ADAMTS-4 (Phe213-Pro431) (Calbiochem, San Diego, CA, US) (final concentration: $0.013 \mu \mathrm{g} / \mu \mathrm{L}$ ), recombinant human ADAMTS1 (R\&D system, 2197-AD-020) (final concentration: $0.015 \mu \mathrm{g} / \mu \mathrm{L}$ ) or $1 \mu \mathrm{L}$ purified recombinant human ADAMTS-5 (CHEMICON, Temecula, CA, USA) (final concentration: $0.015 \mu \mathrm{g} / \mu \mathrm{L}$ ), in a reaction buffer (50 mM Tris, $100 \mathrm{mM}$ $\mathrm{NaCl}, 10 \mathrm{mM} \mathrm{CaCl}$, $\mathrm{pH} 7.5$ ) for $24 \mathrm{~h}$ at $37{ }^{\circ} \mathrm{C}$. Recombinant matrilin-2 was also purified from conditioned medium of cells transfected with matrilin-2 cDNA using Ni-NTA agarose (Qiagen, $\mathrm{GmbH}$, Hilden, Germany). $5 \mu \mathrm{L}$ of purified MATN2 recombinant protein was incubated with $2 \mu \mathrm{L}$ purified recombinant ADAMTS-4 (Calbiochem) at $37{ }^{\circ} \mathrm{C}$ for $24 \mathrm{~h}$. The incubation mixture was separated by SDS-PAGE electrophoresis and analyzed by western blot using the anti-FLAG polyclonal 
antibody and the anti-His monoclonal antibody. The incubation mixture was also analyzed by SELDI mass spectrometry as described previously [43].

\section{Conclusions}

The unique domain of matrilin-2 connects the $C$-terminal coiled-coil oligomerization domain with the protein binding VWA domain arms of the bouquet-shaped molecule. We have identified two proteolytic sites within the unique domain in matrilin-2. The cleavage products were present both in vitro and in vivo. We demonstrated that ADAMTS-4 (aggrecanase-1) and ADAMTS-5 (aggrecanase-2), but not ADAMTS-1, were capable of cleaving matrilin-2. ADAMTS-4 cleaved matrilin-2 not only at the $\mathrm{N}$-terminus of the coiled-coil domain (as in other matrilin proteolytic sites), but also at a previously unreported site between $\mathrm{Asp}^{851}$ and Leu ${ }^{852}$.

\section{Acknowledgments}

This study was supported by grants from NIH (P20GM104937) and Arthritis Foundation to Q. Chen and Roger Williams Hospital Research Funding for S. Iwamoto. We thank Carl R. Flannery for providing the ADAMTS-4 antibody. The authors also thank Lei Wei for his suggestion.

\section{Author Contributions}

ZW carried out the experiment design, molecular genetic studies, protease inhibition and proteolytic cleavage studies, drafted the manuscript. JL cloned three matrilin-2 constructs and found the proteolysis in the transfected COS-1 cell. SI drafted and finalized the manuscript. QC participated in the experiment design and drafted the manuscript. All authors read and approved the final manuscript.

\section{Conflicts of Interest}

The authors declare no conflict of interest.

\section{References}

1. Deak, F.; Wagener, R.; Kiss, I.; Paulsson, M. The matrilins: A novel family of oligomeric extracellular matrix proteins. Matrix Biol. 1999, 18, 55-64.

2. Wagener, R.; Ehlen, H.W.; Ko, Y.P.; Kobbe, B.; Mann, H.H.; Sengle, G.; Paulsson, M. The matrilins-adaptor proteins in the extracellular matrix. FEBS Lett. 2005, 579, 3323-3329.

3. Chen, Q.; Zhang, Y.; Johnson, D.M.; Goetinck, P.F. Assembly of a novel cartilage matrix protein filamentous network: Molecular basis of differential requirement of von Willebrand factor A domains. Mol. Biol. Cell 1999, 10, 2149-2162.

4. Wagener, R.; Kobbe, B.; Paulsson, M. Primary structure of matrilin-3, a new member of a family of extracellular matrix proteins related to cartilage matrix protein (matrilin-1) and von Willebrand factor. FEBS Lett. 1997, 413, 129-134.

5. Belluoccio, D.; Schenkera, T.; Baicib, A.; Trueba, B. Characterization of human matrilin-3 (MATN3). Genomics 1998, 53, 391-439. 
6. Deak, F.; Piecha, D.; Bachrati, C.; Paulsson, M.; Kiss, I. Primary structure and expression of matrilin-2, the closest relative of cartilage matrix protein within the von Willebrand factor type A-like module superfamily. J. Biol. Chem. 1997, 272, 9268-9274.

7. Klatt, A.R.; Nitsche, D.P.; Kobbe, B.; Macht, M.; Paulsson, M.; Wagener, R. Molecular structure, processing, and tissue distribution of matrilin-4. J. Biol. Chem. 2001, 276, 17267-17275.

8. Wagener, R.; Kobbe, B.; Paulsson, M. Matrilin-4, a new member of the matrilin family of extracellular matrix proteins. FEBS Lett. 1998, 436, 123-127.

9. Klatt, A.R.; Paulsson, M.; Wagener, R. Expression of matrilins during maturation of mouse skeletal tissues. Matrix Biol. 2002, 21, 289-296.

10. Klatt, A.R.; Becker, A.K.; Neacsu, C.D.; Paulsson, M.; Wagener, R. The matrilins: Modulators of extracellular matrix assembly. Int. J. Biochem. Cell Biol. 2011, 43, 320-330.

11. Piecha, D.; Wiberg, C.; Mörgelin, M.; Reinhardt, D.P.; Deák, F.; Maurer, P.; Paulsson, M. Matrilin-2 interacts with itself and with other extracellular matrix proteins. Biochem. J. 2002, 367, 715-721.

12. Malin, D.; Sonnenberg-Riethmacher, E.; Guseva, D.; Wagener, R.; Aszódi, A.; Irintchev, A.; Riethmacher, D. The extracellular-matrix protein matrilin 2 participates in peripheral nerve regeneration. J. Cell Sci. 2009, 122, 995-1004.

13. Piecha, D.; Hartmann, K.; Kobbe, B.; Haase, I.; Mauch, C.; Krieg, T.; Paulsson, M. Expression of matrilin-2 in human skin. J. Investig. Dermatol. 2002, 119, 38-43.

14. Ichikawa, T.; Suenaga, Y.; Koda, T.; Ozaki, T.; Nakagawara, A. DeltaNp63/BMP-7-dependent expression of matrilin-2 is involved in keratinocyte migration in response to wounding. Biochem. Biophys. Res. Commun. 2008, 369, 994-1000.

15. Tortorella, M.D.; Burn, T.C.; Pratta, M.A.; Abbaszade, I.; Hollis, J.M.; Liu, R.; Rosenfeld, S.A.; Copeland, R.A.; Decicco, C.P.; Wynn, R.; et al. Purification and cloning of aggrecanase-1: A member of the ADAMTS family of proteins. Science 1999, 284, 1664-1666.

16. Tortorella, M.D.; Pratta, M.; Liu, R.Q.; Austin, J.; Ross, O.H.; Abbaszade, I.; Burn, T.; Arner, E. Sites of aggrecan cleavage by recombinant human aggrecanase-1 (ADAMTS-4). J Biol. Chem. 2000, 275, 18566-18573.

17. Curtis, C.L.; Rees, S.G.; Little, C.B.; Flannery, C.R.; Hughes, C.E.; Wilson, C.; Dent, C.M.; Otterness, I.G.; Harwood, J.L.; Caterson, B.; et al. Pathologic indicators of degradation and inflammation in human osteoarthritic cartilage are abrogated by exposure to n-3 fatty acids. Arthritis Rheumatol. 2002, 46, 1544-1553.

18. Arner, E.C. Aggrecanase-mediated cartilage degradation. Curr. Opin. Pharmacol. 2002, 2, 322-329.

19. Sandy, J.D.; Westling, J.; Kenagy, R.D.; Iruela-Arispe, M.L.; Verscharen, C.; Rodriguez-Mazaneque, J.C.; Zimmermann, D.R.; Lemire, J.M.; Fischer, J.W.; Wight, T.N.; et al. Versican V1 proteolysis in human aorta in vivo occurs at the Glu441-Ala442 bond, a site that is cleaved by recombinant ADAMTS-1 and ADAMTS-4. J. Biol. Chem. 2001, 276, 13372-13378.

20. Flannery, C.R.; Zeng, W.; Corcoran, C.; Collins-Racie, L.A.; Chockalingam, P.S.; Hebert, T.; Mackie, S.A.; McDonagh, T.; Crawford, T.K.; Tomkinson, K.N.; et al. Autocatalytic cleavage of ADAMTS-4 (Aggrecanase-1) reveals multiple glycosaminoglycan-binding sites. J. Biol. Chem. 2002, 277, 42775-42780. 
21. Tortorella, M.; Pratta, M.; Liu, R.-Q.; Abbaszade, I.; Ross, H.; Burn, T.; Arner, E. The thrombospondin motif of aggrecanase-1 (ADAMTS-4) is critical for aggrecan substrate recognition and cleavage. J. Biol. Chem. 2000, 275, 25791-25797.

22. Makihira, S.; Yan, W.; Ohno, S.; Kawamoto, T.; Fujimoto, K.; Okimura, A.; Yoshida, E.; Noshiro, M.; Hamada, T.; Kato, Y.; et al. Enhancement of cell adhesion and spreading by a cartilage-specific noncollagenous protein, cartilage matrix protein (CMP/Matrilin-1), via integrin alpha1beta1. J. Biol. Chem. 1999, 274, 11417-11423.

23. Winterbottom, N.; Tondravi, M.M.; Harrington, T.L.; Klier, F.G.; Vertel, B.M.; Goetinck, P.F. Cartilage matrix protein is a component of the collagen fibril of cartilage. Dev. Dyn. 1992, 193, 266-276.

24. Chen, Q.; Johnson, D.M.; Haudenschild, D.R.; Tondravi, M.M.; Goetinck, P.F. Cartilage matrix protein forms a type II collagen-independent filamentous network: Analysis in primary cell cultures with a retrovirus expression system. Mol. Biol. Cell 1995, 6, 1743-1753.

25. Paulsson, M.; Heinegard, D. Matrix proteins bound to associatively prepared proteoglycans from bovine cartilage. Biochem. J. 1979, 183, 539-545.

26. Wiberg, C.; Klatt, A.R.; Wagener, R.; Paulsson, M.; Bateman, J.F.; Heinegård, D.; Mörgelin, M. Complexes of matrilin-1 and biglycan or decorin connect collagen VI microfibrils to both collagen II and aggrecan. J. Biol. Chem. 2003, 278, 37698-37704.

27. Wu, J.J.; Eyre, D.R. Matrilin-3 forms disulfide-linked oligomers with matrilin-1 in bovine epiphyseal cartilage. J. Biol. Chem. 1998, 273, 17433-17438.

28. Zhang, Y.; Chen, Q. Changes of matrilin forms during endochondral ossification. Molecular basis of oligomeric assembly. J. Biol. Chem. 2000, 275, 32628-32634.

29. Zhang, Y.; Wang, Z.-K.; Luo, J.-M.; Kanbe, K.; Chen, Q. Multiple functions of the von Willebrand Factor A domain in matrilins: Secretion, assembly, and proteolysis. J. Orthop. Surg. Res. 2008, 3, 21.

30. Hills, R.; Mazzarella, R.; Fok, K.; Liu, M.; Nemirovskiy, O.; Leone, J.; Zack, M.D.; Arner, E.C.; Viswanathan, M.; Abujoub, A.; et al. Identification of an ADAMTS-4 cleavage motif using phage display leads to the development of fluorogenic peptide substrates and reveals matrilin-3 as a novel substrate. J. Biol. Chem. 2007, 282, 11101-11109.

31. Ehlen, H.W.; Sengle, G.; Klatt, A.R.; Talke, A.; Müller, S.; Paulsson, M.; Wagener, R. Proteolytic processing causes extensive heterogeneity of tissue matrilin forms. J. Biol. Chem. 2009, 284, 21545-21556.

32. Li, L.; Zhang, L.; Shao, Y.; Wang, G.; Gong, R.; Wang, Z.; Peng, J.; Wang, S.; Genochio, D.; Zhao, B.; et al. Distinct roles of two alternative splice variants of matrilin-2 in protein oligomerization and proteolysis. Mol. Med. Rep. 2012, 6, 1204-1210.

33. Hughes, C.E.; Little, C.B.; Büttner, F.H.; Bartnik, E.; Caterson, B. Differential expression of aggrecanase and matrix metalloproteinase activity in chondrocytes isolated from bovine and porcine articular cartilage. J. Biol. Chem. 1998, 273, 30576-30582.

34. Nakamura, H.; Fujii, Y.; Inoki, I.; Sugimoto, K.; Tanzawa, K.; Matsuki, H.; Miura, R.; Yamaguchi, Y.; Okada, Y. Brevican is degraded by matrix metalloproteinases and aggrecanase-1 (ADAMTS4) at different sites. J. Biol. Chem. 2000, 275, 38885-38890. 
35. Westling, J.; Gottschall, P.E.; Thompson, V.P.; Cockburn, A.; Perides, G.; Zimmermann, D.R.; Sandy, J.D. ADAMTS4 (aggrecanase-1) cleaves human brain versican V2 at Glu405-Gln406 to generate glial hyaluronate binding protein. Biochem. J. 2004, 377, 787-795.

36. Matthews, R.T.; Gary, S.C.; Zerillo, C.; Pratta, M.; Solomon, K.; Arner, E.C.; Hockfield, S. Brain-enriched hyaluronan binding (BEHAB)/brevican cleavage in a glioma cell line is mediated by a disintegrin and metalloproteinase with thrombospondin motifs (ADAMTS) family member. J. Biol. Chem. 2000, 275, 22695-22703.

37. Tortorella, M.D.; Arner, E.C.; Hills, R.; Easton, A.; Korte-Sarfaty, J.; Fok, K.; Wittwer, A.J.; Liu, R.Q.; Malfait, A.M. $\alpha$-Macroglobulin is a novel substrate for ADAMTS-4 and ADAMTS-5 and represents an endogenous inhibitor of these enzymes. J. Biol. Chem. 2004, 279, 17554-17561.

38. Westling, J.; Fosang, A.J.; Last, K.; Thompson, V.P.; Tomkinson, K.N.; Hebert, T.; McDonagh, T.; Collins-Racie, L.A.; LaVallie, E.R.; Morris, E.A.; et al. ADAMTS4 cleaves at the aggrecanase site (Glu373-Ala374) and secondarily at the matrix metalloproteinase site (Asn341-Phe342) in the aggrecan interglobular domain. J. Biol. Chem. 2002, 277, 16059-16066.

39. Piecha, D.; Kiss, I.; Paulsson, M.; Deák, F.; Hauser, N.; Studer, D.; Muratoglu, S.; Mörgelin, M. Matrilin-2, a large, oligomeric matrix protein, is expressed by a great variety of cells and forms fibrillar networks. J. Biol. Chem. 1999, 274, 13353-13361.

40. Jayasuriya, C.T.; Goldring, M.B.; Terek, R.; Chen, Q. Matrilin-3 induction of IL-1 receptor antagonist Is required for up-regulating collagen II and aggrecan and down-regulating ADAMTS-5 gene expression. Arthritis Res. Ther. 2012, doi:10.1186/ar4033.

41. Lefebvre, V.; Garofalo, S.; de Crombrugghe, B. Type X collagen gene expression in mouse chondrocytes immortalized by a temperature-sensitive simian virus 40 large tumor antigen. J. Cell Biol. 1995, 128, 239-245.

42. Chen, Q.; Johnson, D.M.; Haudenschild, D.R.; Goetinck, P.F. Progression and recapitulation of the chondrocyte differentiation program: Cartilage matrix protein is a marker for cartilage maturation. Dev. Biol. 1995, 172, 293-306.

43. Zhang, Y.; Chen, Q. The noncollagenous domain 1 of type X collagen. A novel motif for trimer and higher order multimer formation without a triple helix. J. Biol. Chem. 1999, 274, 22409-22413.

Sample Availability: Samples of the compounds and enzymes are ordered from the companies and are not available from the authors.

(C) 2014 by the authors; licensee MDPI, Basel, Switzerland. This article is an open access article distributed under the terms and conditions of the Creative Commons Attribution license (http://creativecommons.org/licenses/by/3.0/). 